\title{
RISK OF EARLY \& LATE OBSTETRIC COMPLICATIONS IN WOMEN WITH IVF- CONCEIVED PREGNANCIES AND POLYCYSTIC OVARY SYNDROME (PCOS)
}

\author{
N. A. Bagegni, BS ${ }^{1}$, J Blaine, BS ${ }^{1}$, B J VanVoorhis, MD ${ }^{1}$, A Dokras, MD.PhD ${ }^{2}$
}

Key words: PCOS, pregnancy, gestational diabetes, obesity, miscarriage

\section{ABSTRACT}

There is conflicting evidence on the association between PCOS and early and late obstetric complications. It is unclear if the reported risks are independent of BMI, preexisting hypertension and diabetes. We performed a retrospective chart review to examine the risk of early and late obstetrical complications after IVF in women with PCOS $(n=130)$ compared to controls $(n=130)$. The miscarriage rate was $17.7 \%$ in PCOS women and $15.4 \%$ in controls. PCOS was not associated with miscarriage independent of age and BMI. The prevalence of gestational DM (GDM) was similar in both groups (12\% PCOS versus $11 \%$ controls). BMI was independently associated with GDM $(p=0.01)$. Risk of both preeclampsia and $\mathrm{PIH}$ was $10 \%$ in PCOS and $5 \%$ in controls, but not statistically significant. Preexisting HTN showed a significant association with preeclampsia $(p<0.01)$. There was no significant difference in preterm delivery, twin gestation, intrauterine fetal death and intrauterine growth restriction in the
\end{abstract}

2 groups. Our study did not detect an increased risk of miscarriage in women with PCOS undergoing IVF. Obesity was a significant contributor to late obstetric complications, namely GDM. These findings warrant counseling of women with PCOS especially prior to IVF on the potential benefits of weight loss prior to pregnancy.

${ }^{1}$ Department of Obstetrics and Gynecology, University of lowa, lowa City, USA 52242 and ${ }^{2}$ University of Pennsylvania, Philadelphia, USA 19104.

\section{Summary for Lay Readers}

There is conflicting evidence on the association between polycystic ovary syndrome (PCOS) and early and late pregnancy complications. It is unclear if the reported risks are independent of maternal weight and medical conditions such as preexisting hypertension and diabetes. We performed a study to examine the risk of early and late pregnancy complications after in vitro fertilization in a large group of women with PCOS $(n=130)$ compared to controls $(n=130)$. The miscarriage rate

Please cite this paper as: Bagegni NA, Blaine J, VanVoorhis BJ, Dokras A. Risk of Early \& Late Complications in Women with Polycystic Ovary Syndrome (PCOS). Proc Obstet Gynecol. 2010 Oct;1(2): Article 2 [10 p.]. Available from: http://ir.uiowa.edu/pog/vol1/iss2/2/. Free full text article.

Corresponding Author: Anuja Dokras, MD.,PhD, Department of Obstetrics and Gynecology, University of Pennsylvania, 3701 Market Street, Suite 800, Philadelphia, PA 19104., Telephone (215)615-0085, (FAX)(215)3495512, adokras@obgyn.upenn.edu

This is an Open Access article distributed under the terms of the Creative Commons Attribution 3.0 Unported License (http://creativecommons.org/licenses/by/3.0), which permits unrestricted use, distribution, and reproduction in any medium, provided the original work is properly cited. 
was similar in PCOS women and controls. PCOS was not associated with miscarriage independent of age and BMI. The prevalence of gestational DM (GDM) was also similar in both groups (12\% PCOS versus $11 \%$ controls). PCOS was not associated with GDM, however, maternal weight was significantly associated with GDM. Risk of both pre-eclampsia and pregnancy induced hypertension was $10 \%$ in PCOS and $5 \%$ in controls, but not statistically significant. Women with preexisting hypertension had a higher risk of developing preeclampsia. There was no significant difference in preterm delivery, Cesarean section, twin gestation, intrauterine fetal death and intrauterine growth restriction in the 2 groups. Our study shows that obesity was a significant contributor to pregnancy complications such as gestational diabetes. These findings may warrant aggressive counseling of women with PCOS on the potential benefits of weight loss prior to pregnancy.

\section{INTRODUCTION}

Polycystic ovary syndrome (PCOS) is one of the most common endocrine disorders affecting women of childbearing age, with an estimated prevalence of about $5-10 \%$ as reported in population-based studies ${ }^{1}$. PCOS is a chronic disorder associated with both reproductive complications and early onset metabolic morbidities ${ }^{2}$. Women with PCOS can be very successful in achieving a pregnancy after treatment with ovulation induction drugs $^{3}$. The increased prevalence of metabolic abnormalities associated with PCOS however, may have lifelong health implications ${ }^{2}$. Several studies in diverse populations of PCOS women have demonstrated an increased risk of hyperinsulinemia ${ }^{2}$, insulin resistance, dyslipidemia $^{4}$, glucose intolerance ${ }^{5}$, hypertension ${ }^{2}$ and metabolic syndrome ${ }^{6}$. Over $50 \%$ of women with PCOS are either overweight or obese further modifying this metabolic phenotype ${ }^{7}$. We have recently reported that obesity itself is associated with adverse reproductive and obstetric outcomes ${ }^{8}$. In addition, pregnancy is characterized as a state of insulin resistance ${ }^{9}$. The metabolic risks of PCOS may therefore adversely affect both maternal and fetal status, potentially leading to an increased manifestation of complications such as gestational diabetes mellitus (GDM), pregnancy induced hypertension (PIH) and preeclampsia.

There is conflicting evidence on the association between PCOS and early and late obstetric complications. A few studies have reported an increased risk of obstetric and neonatal complications in women with $\mathrm{PCOS}^{10}$. Limitations of some of these studies include small study sizes, differing diagnostic criteria for PCOS and heterogeneity in controlling for variables such as maternal BMI, parity, preexisting diabetes and hypertension. It is therefore not clear if PCOS per se increases the risk of antepartum complications or if the presence of preexisting metabolic abnormalities increases the overall obstetric risks in PCOS.

Our primary aim was to determine the risk of early obstetric complications such as spontaneous miscarriage and late complications such as GDM, PIH, preeclampsia and preterm delivery (PTD) in a large group of women with PCOS compared to controls. Our secondary aim was to determine the effects of PCOS on neonatal outcomes. 


\section{METHODS}

We reviewed pregnancy outcomes in women with PCOS $(n=130)$ and controls (women with tubal factor infertility, $\mathrm{n}=130$ ) who had in vitro fertilization (IVF) between 1995 and 2007 at the University of lowa Hospitals \& Clinics. Subjects who had a diagnosis of PCOS based on the 2003 Rotterdam criteria (presence of at least two of the following three criteria: oligomenorrhea $\quad(<9$ cycles/year), clinical and/or biochemical signs of hyperandrogenism, and polycystic ovaries on ultrasound) were included in the study ${ }^{11}$. Subjects who had a two step approach to screening for gestational diabetes were included in the study. An initial screen was performed by measuring the 1 hour glucose level after a $50 \mathrm{~g}$ oral glucose load. The 3 hour oral glucose tolerance test (GTT) was performed if the screening level was $>140 \mathrm{mg} / \mathrm{dl}$. Gestational diabetes mellitus (GDM) was defined as two or more abnormal blood glucose levels on a 3 hour GTT (fasting $\geq 95 \mathrm{mg} / \mathrm{dl}$, $1 \mathrm{hr}$ level $\geq 180 \mathrm{mg} / \mathrm{dl}$, $2 \mathrm{hr}$ level $\geq 155 \mathrm{mg} / \mathrm{dl}$, $3 \mathrm{hr}$ level $\geq$ $140 \mathrm{mg} / \mathrm{dl})^{12}$. Pregnancy induced hypertension (PIH) was defined as blood pressure $\geq 140 / 90 \mathrm{mmHg}$ after 20 weeks gestation without proteinuria and with normal blood pressure prior to pregnancy. Preeclampsia was defined as blood pressure $\geq 140 / 90 \mathrm{mmHg}$ after 20 weeks gestation and proteinuria ( $>300 \mathrm{mg}$ ) determined on a 24 hour urine collection. This information was abstracted from the medical records and IVF database. Subjects with incomplete obstetric records were excluded from the study. Control subjects with only tubal factor infertility were selected consecutively from the IVF database to match the similar time period, age $(+/-5$ years) and BMI (+/-2) of the PCOS subjects.
Other variables that were compared included gravidity, parity, current smoking, pre-existing diabetes, hypertension and use of medications to treat these latter conditions. Prior to the current pregnancy impaired fasting glucose (IFG) was defined as having a fasting blood glucose level of 100$125 \mathrm{mg} / \mathrm{dL}$ and diabetes was defined as glucose $\geq 126 \mathrm{mg} / \mathrm{dl}$. For subjects who had a $2 \mathrm{~h}$ GTT, impaired glucose tolerance (IGT) was defined as 2 hour glucose level of $140-199 \mathrm{mg} / \mathrm{dl}$ and diabetes was defined as glucose $\geq 200 \mathrm{mg} / \mathrm{dl}$. Antepartum outcomes analyzed included miscarriage rate (loss before 20 weeks gestation), GDM, PIH, preeclampsia, preterm delivery (PTD $<37$ weeks), intrauterine growth restriction (IUGR,) and intrauterine fetal demise (IUFD). Intrapartum and neonatal outcomes were also compared between control and study groups.

The Wilcoxon rank sum test was used to evaluate differences between continuous variables and the Fisher exact test and Chi-squared test was performed for categorical variables to compare demographic data between the PCOS group and controls. Multiplelogistic regression analysis was performed to control for variables that significantly differed in the two groups. Statistical significance was defined as $p$ $<0.05$. We had $80 \%$ power to detect a 2 fold difference in miscarriage rates between women with PCOS and controls at the 0.05 significance level. This study was approved by the University of lowa Institutional Review Board. 


\section{RESULTS}

\section{Demographics}

Women with PCOS were slightly younger (mean age $31.1 \pm 4$.3years than controls $33 \pm 3.7 y e a r s$ ), and had a higher prepregnancy BMI compared to the controls (mean BMI $31 \pm 8$ vs. controls $28.9 \pm 5.5, p<0.05$, Table 1). The PCOS subjects had fewer prior pregnancies and miscarriages than the controls. However, women with PCOS had a higher miscarriage rate per pregnancy $50 \%(42 / 85)$ compared to that in controls $24 \%(81 / 341, p<0.01)$. Similar proportions of women with PCOS and controls had preexisting hypertension $(10 \%$ vs $6 \%)$ and hypothyroidism $(10 \%$ vs $7 \%$ ). In the PCOS group $29 \%$ had IFG/IGT and $5 \%$ had type 2 diabetes. A higher proportion of women with PCOS had taken insulin sensitizing agents, namely metformin, prior to the current pregnancy compared to controls $(23.9 \%$ vs $0.8 \%$ ). All 5 subjects with DM, $9 / 30$ subjects with IFG/IGT and 14 other subjects were prescribed metformin. The majority of women with PCOS $(54 \%)$ met all three diagnostic criteria namely oligomenorrhea, hyperandrogenism and polycystic ovaries.

Table 1. Demographic characteristics of women with PCOS and Controls

\begin{tabular}{|l|c|c|}
\hline Variable & PCOS (n=130) & Controls (n=130) \\
\hline Mean age in years (SD) & $31.1(4.3)$ & $33.0(3.7)^{\star *}$ \\
\hline Mean prepregnancy BMI, (SD) & $31(8)$ & $28.9(5.5)^{*}$ \\
\hline Duration of infertility in months (SD) & $45.1(2.7)$ & $46.9(3.9)$ \\
\hline Nulligravid & $78(60 \%)$ & $20(15 \%)^{* *}$ \\
\hline Previous miscarriage & & \\
0 & $98(75 \%)$ & $81(62 \%)^{*}$ \\
1 & $25(19 \%)$ & $30(23 \%)$ \\
2 & $7(5 \%)$ & $19(15 \%)$ \\
\hline Race & $87 \%$ & $88 \%$ \\
Caucasian & $3 \%$ & $1 \%$ \\
Hispanic & $2 \%$ & $3 \%$ \\
African American & $2 \%$ & $1 \%$ \\
Asian & $0 \%$ & $6 \%$ \\
Other & $6 \%$ & $12(10 \%)^{*}$ \\
Unknown & $4(3 \%)$ & $15 \mathrm{missing}$ \\
\hline Smoking: Current & 2 missing & \\
& & \\
& &
\end{tabular}




\section{Early Obstetric Complications}

Miscarriage rate: The miscarriage rate (all spontaneous losses $<20$ weeks) in women with PCOS was $17.7 \%(23 / 130)$ compared to $15.4 \%(20 / 130)$ in controls (Table 2). A higher number of PCOS subjects had second trimester miscarriages compared to controls but this was not statistically significant. In addition, seven twin pregnancies spontaneously reduced to singletons in the PCOS group compared to 14 twin pregnancies in the control group. Two PCOS subjects with diabetes $(2 / 5)$ and 5 PCOS subjects with IGT/IFG (5/30) had a miscarriage. PCOS was not found to be significantly associated with miscarriage $(p=0.495)$ after adjusting for age, $\mathrm{BMI}$, and previous miscarriage in a logistic regression model. Age appeared to have a weak association with miscarriage rate (OR1.48, 95\% Cl: 0.96 , 2.26, $\mathrm{p}<0.07$ ).

\section{Late Obstetric complications}

Gestational Diabetes: The percentage of women with ongoing pregnancy beyond 20 weeks who developed GDM was not significantly different in the two groups, PCOS $12.2 \% \quad(13 / 102)$ and controls $10.9 \%(12 / 109) \quad(p=0.78$, Table 2). The subjects with preexisting diabetes (PCOS 5, controls 1) were not included in this analysis. The logistic regression model for GDM with PCOS, age, and $\mathrm{BMI}$ as independent variables showed that BMI was significantly associated with GDM after adjusting for age and diagnosis of PCOS (OR 1.44, $95 \% \mathrm{Cl}: 1.09-1.92$, p<0.01). PCOS was not found to be significantly associated with GDM $(p=0.678)$ after adjusting for age and BMI. PCOS women with singletons $(n=73)$ had a $15.1 \%$ prevalence of GDM compared to $13.2 \%$ in controls with singletons $(n=76$, Table 3 ). The rate of multiple pregnancy was similar in both groups, $32 \%$ (34) in PCOS and $31 \%$ (29) in controls (Table $3)$. Of the 25 PCOS women with IFG/IGT with ongoing pregnancies, 5 developed GDM (20\%) and three of the latter women had been treated with metformin prior to pregnancy.

\section{Pregnancy induced hypertension/preeclampsia: The} prevalence of $\mathrm{PIH}$ was two times higher in women with PCOS than that of controls $(10.28 \%$ vs. $5.45 \%, p=0.186$, Table II) as was the prevalence of preeclampsia (PCOS 10.28\% compared to controls $5.45 \%$, Table 2). The association between PCOS and $\mathrm{PIH}$ after adjusting for the effect of smoking was not significant $(p=0.073)$. Preexisting hypertension had a significant association with development of preeclampsia in both groups (OR 5.02, 95\% Cl: 1.39-18.16, p<0.01). Also, nulligravidity had a strong association with the development of preeclampsia in both groups (OR 7.30, $955 \mathrm{Cl}$ : 1.97 , $27.04, p<0.003)$. PCOS was not found to be significantly associated with preeclampsia after adjusting for any of the above covariates.

Preterm Delivery: The overall risk of PTD was $26 \%$ in women with PCOS compared to $26 \%$ in controls $(p=0.9$, Table 2). PCOS was not found to be significantly associated with PTD after adjusting for multiple pregnancy, age, $\mathrm{BMI}$, and parity $(\mathrm{p}=0.6)$. Twin gestation had a significantly higher risk of PTD in the PCOS and control groups (OR 13.31, 95\% Cl: 5.98-29.6, $p<0.001$, Table 3). In addition, BMI also had a significant association with PTD (OR 1.34, $955 \mathrm{Cl}$ : 1.03-1.76, $\mathrm{p}<0.03$ ). 
Table 2. Obstetric complications in women with PCOS and Controls

\begin{tabular}{|l|c|c|}
\hline Variable & PCos & Controls \\
\hline $\begin{array}{l}\text { Miscarriage } \\
<12 \text { weeks } \\
12-20 \text { weeks }\end{array}$ & $\begin{array}{c}23(17.7 \%) \\
17 \\
6\end{array}$ & $\begin{array}{c}20(15.4 \%) \\
19 \\
1\end{array}$ \\
\hline Twin gestation & $34(32 \%)$ & $29(31 \%)$ \\
\hline Gestational Diabetes (GDM) & $13(12.2 \%)$ & $12(10.9 \%)$ \\
\hline $\begin{array}{l}\text { Pregnancy induced } \\
\text { hypertension(PIH) }\end{array}$ & $11(10.3 \%)$ & $6(5.5 \%)$ \\
\hline Preeclampsia & $11(10.3 \%)$ & $6(5.5 \%)$ \\
\hline $\begin{array}{l}\text { Preterm delivery } \\
\text { PTD) }\end{array}$ & $26(24 \%)$ & $26(24 \%)$ \\
\hline Intrauterine fetal death(IUFD) & 0 & $1(0.9 \%)$ \\
\hline Intrauterine growth restriction(IUGR) & $2(2 \%)$ & $4(4 \%)$ \\
\hline
\end{tabular}

Table 3. Obstetric Complications: Singleton compared to Twin gestation in women with PCOS and controls

\begin{tabular}{|l|l|l|l|l|}
\hline & \multicolumn{2}{|c|}{ PCOS } & \multicolumn{2}{c|}{ Controls } \\
\hline Variable & $\begin{array}{l}\text { Singleton } \\
(n=73)\end{array}$ & $\begin{array}{l}\text { Twin } \\
(n=34)\end{array}$ & $\begin{array}{l}\text { Singleton } \\
(n=76)\end{array}$ & $\begin{array}{l}\text { Twin } \\
(n=29)\end{array}$ \\
\hline Gestational Diabetes (GDM) & $11(15.1 \%)$ & $2(5.9 \%)$ & $10(13.2 \%)$ & $2(5.9 \%)$ \\
\hline $\begin{array}{l}\text { Pregnancy induced hypertension } \\
(P I H)\end{array}$ & $7(9.6 \%)$ & $4(11.8 \%)$ & $6(7.9 \%)$ & $0(0 \%)$ \\
\hline Preeclampsia & $7(9.6 \%)$ & $4(11.8 \%)$ & $3(3.9 \%)$ & $3(8.8 \%)$ \\
\hline Preterm delivery (PTL) & $6(8.2 \%)$ & $20(58.8 \%)$ & $8(10.5 \%)$ & $18(62 \%)$ \\
\hline IUGR & $1(1.4 \%)$ & $1(2.9 \%)$ & $3(3.9 \%)$ & $1(2.9 \%)$ \\
\hline
\end{tabular}

Neonatal \& Intrapartum Outcomes: Women with PCOS had a total of 107 deliveries and controls had 110 deliveries. There was no significant difference in delivery mode in either group, with approximately $44 \%$ women delivering by cesarean section (Table 4). The mean birth weights of both singletons and twin gestation did not differ between groups and the incidence of macrosomia (birth weight $>4000 \mathrm{~g}$ ) was not statistically different in infants of 
controls compared to infants of women with PCOS. The risk of IUGR was higher in the control group compared to the PCOS group, however the numbers in each group were small and not significantly different $(p=0.68)$.

Table 4. Neonatal \& Intrapartum Outcomes in women with PCOS compared to Controls

\begin{tabular}{|l|l|l|}
\hline Variable & PCOS (n=107) & Controls (n=110) \\
\hline $\begin{array}{l}\text { Delivery Mode } \\
\text { C-Section }\end{array}$ & $48(45 \%)$ & $47(43 \%)$ \\
\hline $\begin{array}{l}\text { Birth Weight, mean(SD) } \\
\text { Singleton }(g) \\
\text { Twins (g) }\end{array}$ & $\begin{array}{l}3383(681) \\
2376(796)\end{array}$ & $\begin{array}{l}3384(771) \\
2474(639)\end{array}$ \\
\hline $\begin{array}{c}\text { Macrosomia (>4,000 g) } \\
\text { Infant Gender } \\
\text { Male }\end{array}$ & $10(6.9 \%)$ & $12(8.3 \%)$ \\
\hline
\end{tabular}

\section{DISCUSSION}

Women with PCOS need to be accurately counseled regarding their obstetric risks especially if some of these risks can be modified by interventions such as weight loss. We included a large group of well defined subjects with PCOS undergoing IVF and attempted to match them based on key factors such as age and BMI. After using logistic regression for confounding factors our study did not show an increased risk of miscarriage in women with PCOS independent of BMI. This supports previous data from a metaanalysis of nine studies that reported no significant difference in miscarriage rates between PCOS and non-PCOS patients after IVF (random effects OR $1.0 ; 95 \% \mathrm{Cl} 0.5$ to 1.8$)^{13}$. The results of the meta-analysis were however limited in that not all studies were matched for age or BMI and most included multiple cycles from one subject. Regardless of the method of conception women with a BMI $\geq 25$ have been shown to have a significantly higher odds of miscarriage (OR 1.67; 95\% Cl, 1.25-2.25) $)^{14}$. In IVFconceived pregnancies, women with a $\mathrm{BMI} \geq 30$ had a higher risk of miscarriage compared to women with a $\mathrm{BMI}<30$ (OR 1.53, 95\% Cl 1.27-1.84 (p $<0.001)^{15}$. The current literature indicates that, regardless of the method of conception, miscarriage risk is related to obesity rather than PCOS or any other cause of infertility.

Higher multiple pregnancy rate, parity and BMI in women with PCOS were important risks contributing to the development of $\mathrm{GDM}^{10}$. Our study showed that BMI was an independent risk factor for the development of GDM after controlling for age and PCOS diagnosis. The independent risk of GDM in women with PCOS has been investigated in several populations but remains controversial. In a Finnish study 
99 women with PCOS (ultrasound appearance of polycystic ovaries and hyperandrogenism, with or without irregular menses) were compared to 737 geographically matched controls ${ }^{16}$. The risk of developing GDM was largely explained by obesity and the independent association with PCOS could not be demonstrated in singleton pregnancies. In a Czech study including PCOS women $(n=66)$ and age and weight matched controls $(n=66)$ no difference was detected in the risk of $\mathrm{GDM}^{17}$. A recent population-based U.S. study reported a higher prevalence of GDM among women with PCOS $(n=1,542)$ compared to controls (OR 2.4, 95\% Cl: 2.1-2.83) ${ }^{18}$. However, despite its large sample size, a major drawback of this study was that BMI was not included in the analysis. A recent metaanalysis of five studies demonstrated a higher risk of developing GDM in women with PCOS (OR 3.66, 95\% Cl: 1.2011.16) however, significant statistical heterogeneity was present in these studies ${ }^{10}$.The risk of developing GDM in all women is reported to be about two, four, and eight times higher based on overweight, obese, and severely obesity respectively, compared with normalweight $^{19}$. Larger studies are therefore needed in a well characterized PCOS population to determine the independent risk of GDM.

Our study suggests a possible association between PCOS and PIH but was limited by sample size. As expected preexisting hypertension and nulliparity was associated with increased risk of preeclampsia. The prevalence of preeclampsia appears to be two-fold greater in women with PCOS, but was not statistically significant in our study. In a recent study of women with PCOS a subgroup analysis of only two higher validity studies was performed and reported a significantly increased risk of PIH (OR $3.71,95 \% \mathrm{Cl}: 1.72-17.49)^{10}$. In the same study women with PCOS also demonstrated a significantly higher chance of developing preeclampsia (OR 3.47, 95\% Cl: 1.95-6.17). However, no subgroup analysis could be performed as variables such as parity, BMI and multiple pregnancy differed in the studies included. As reported in previous studies we did not find an increased risk of PTD delivery independent of multiple gestations in women with $\mathrm{PCOS}^{10}$. Similarly we did not find an increased risk of macrosomia or SGA in children born to women with PCOS. Studies have demonstrated an increased risk of poor obstetric outcomes (preterm delivery, low birth weight) in singleton pregnancies resulting from $\operatorname{IVF}^{20,21}$. Both groups in our study conceived after IVF thereby controlling for any confounding effect.

In summary our study demonstrates that the miscarriage rate in women with PCOS after IVF is not increased independent of BMI. However, BMI alone was significantly associated with increased risk of gestational diabetes. Preexisting hypertension in the PCOS population was associated with an increased risk of preeclampsia. These findings underscore the need for clinicians to diligently screen women with PCOS for the presence of obesity and hypertension. Physicians have an opportunity to screen women prior to assisting conception and offer nutritional counseling and weight loss strategies. Obese women with PCOS should be monitored closely in pregnancy for the development of GDM, PIH and preeclampsia. Accurate identification of these morbidities in pregnancy is critical as the affected group of women may also be at increased risk for hypertension, heart disease and cardiovascular events later in life $22,23$. 
Future studies should aim to examine the effects of interventions such as prepregnancy weight loss on

\section{References}

1. Carmina E, Lobo RA. Polycystic ovary syndrome (PCOS): arguably the most common endocrinopathy is associated with significant morbidity in women. $\mathrm{J}$ Clin Endocrinol Metab. 1999 Jun;84(6):1897-9.

2. Ehrmann DA. Polycystic ovary syndrome. N Engl J Med. 2005 Mar 24;352(12):1223-36.

3. Eijkemans MJ, Imani B, Mulders AG, Habbema JD, Fauser BC. High singleton live birth rate following classical ovulation induction in normogonadotrophic anovulatory infertility (WHO 2). Hum Reprod. 2003 Nov;18(11):2357-62.

4. Wild RA, Painter PC, Coulson PB, Carruth KB, Ranney GB. Lipoprotein lipid concentrations and cardiovascular risk in women with polycystic ovary syndrome. J Clin Endocrinol Metab. 1985 Nov;61(5):946-51.

5. Ehrmann DA, Barnes RB, Rosenfield RL, Cavaghan MK, Imperial J. Prevalence of impaired glucose tolerance and diabetes in women with polycystic ovary syndrome. Diabetes Care. 1999 Jan;22(1):141-6.

6. Dokras A, Bochner M, Hollinrake E, Markham S, Vanvoorhis B, Jagasia $\mathrm{DH}$. Screening women with polycystic ovary syndrome for metabolic syndrome. Obstet Gynecol. 2005 Jul;106(1):131-7. miscarriage rate and risk of GDM in women with PCOS.
7. Carmina E, Legro RS, Stamets K, Lowell J, Lobo RA. Difference in body weight between American and Italian women with polycystic ovary syndrome: influence of the diet. Hum Reprod. 2003 Nov;18(11):2289-93.

8. Dokras A, Baredziak L, Blaine J, Syrop C, VanVoorhis BJ, Sparks A. Obstetric outcomes after in vitro fertilization in obese and morbidly obese women. Obstet Gynecol. $2006 \mathrm{Jul} ; 108(1): 61-9$.

9. Dokras A, Spaczynski RZ, Behrman HR, Duleba AJ. Testosterone levels in pregnant women correlate with the insulin response during the glucose tolerance test. Fertil Steril. 2003 Mar;79(3):492-7.

10. Boomsma CM, Eijkemans MJ, Hughes EG, Visser GH, Fauser $B C$, Macklon NS. A meta-analysis of pregnancy outcomes in women with polycystic ovary syndrome. Hum Reprod Update. 2006 NovDec;12(6):673-83.

11. Rotterdam ESHRE/ASRMSponsored PCOS Consensus Workshop Group. Revised 2003 consensus on diagnostic criteria and long-term health risks related to polycystic ovary syndrome. Fertil Steril. 2004 Jan;81(1):19-25.

12. American Diabetes Association. Diagnosis and classification of diabetes mellitus. Diabetes Care. 2008 Jan;31 Suppl 1:S55-60.

13. Heijnen EM, Eijkemans MJ, Hughes EG, Laven JS, Macklon NS, Fauser BC. A meta-analysis of outcomes of conventional IVF in women with polycystic ovary syndrome. Hum Reprod Update. 2006 Jan-Feb;12(1):13-21. 
14. Metwally M, Ong KJ, Ledger WL, Li TC. Does high body mass index increase the risk of miscarriage after spontaneous and assisted conception? A meta analysis of the evidence. Fertil Steril. 2008 Sep;90(3):714-26.

15. Maheshwari A, Stofberg L, Bhattacharya S. Effect of overweight and obesity on assisted reproductive technology--a systematic review. Hum Reprod Update. 2007 Sep-Oct;13(5):43344.

16. Mikola M, Hiilesmaa V, Halttunen M, Suhonen L, Tiitinen A. Obstetric outcome in women with polycystic ovarian syndrome. Hum Reprod. 2001 Feb;16(2):226-9.

17. Haakova L, Cibula D, Rezabek K, Hill M, Fanta M, Zivny J. Pregnancy outcome in women with PCOS and in controls matched by age and weight. Hum Reprod. 2003 Jul;18(7):1438-41.

18. Lo JC, Feigenbaum SL, Escobar GJ, Yang J, Crites YM, Ferrara A. Increased prevalence of gestational diabetes mellitus among women with diagnosed polycystic ovary syndrome: a population-based study. Diabetes Care. 2006 Aug;29(8):1915-7.

19. Chu SY, Callaghan WM, Kim SY, Schmid CH, Lau J, England LJ, Dietz PM. Maternal obesity and risk of gestational diabetes mellitus. Diabetes Care. 2007 Aug;30(8):2070-6.

20. Helmerhorst FM, Perquin DA, Donker D, Keirse MJ. Perinatal outcome of singletons and twins after assisted conception: a systematic review of controlled studies. BMJ. 2004 Jan 31;328(7434):261-4.
21. Schieve LA, Meikle SF, Ferre C, Peterson HB, Jeng G, Wilcox LS. Low and very low birth weight in infants conceived with use of assisted reproductive technology. N Engl J Med. 2002 Mar 7;346(10):731-7.

22. Germain AM, Romanik MC, Guerra I, Solari S, Reyes MS, Johnson RJ, Price K, Karumanchi SA, Valdés G. Endothelial dysfunction: a link among preeclampsia, recurrent pregnancy loss, and future cardiovascular events? Hypertension. 2007 Jan;49(1):90-5.

23. Agatisa PK, Ness RB, Roberts JM, Costantino JP, Kuller LH, McLaughlin MK. Impairment of endothelial function in women with a history of preeclampsia: an indicator of cardiovascular risk. Am J Physiol Heart Circ Physiol. 2004 Apr;286(4):H1389-93. 